\title{
SUR LA PROLIFẼRATION DANS LA SEINE D'UNE DIATOMÉE BENTHIQUE TROPICALE : NAVICULA CONFERVACEA (KUTZ.) GRUNOW ${ }^{1}$
}

\author{
par M. Coste ${ }^{2}$.
}

\begin{abstract}
Une étude des peuplements benthiques de la Seine portant sur plus de 250 échantillons prélevés sur 39 stations échelonnées entre la source et Rouen, a permis de localiser la prolifération d'une diatomée considérée comme «endémique» des régions tropicales, à l'aval immédiat d'une centrale thermique (E.D.F. Porcheville). La morphologie, l'ultrastructure et la répartition géographique sont examinées succinctement. Les données écologiques recueillies à Porcheville, confrontées à celles de la littérature conduisent à considérer Navicula confervacea comme une espèce saprophyte polluorésistante, sténotherme des eaux chaudes $\left(25-31^{\circ} \mathrm{C}\right)$.
\end{abstract}

\section{The proliferation in the Seine of a tropical benthic diatom : Navicula confervacea (Kütz.) Grunow.}

This study of the benthic populations in the Seine was based on more than 250 samples taken at 39 stations which were regularly distributed between the source and Rouen. It was possible to locate the site at which there was proliferation of a benthic diatom, usually considered as «endemic» to the tropics, and this site was immediately downstream from a thermal power station. The morphology, ultrastructure and geographical distribution of this species are briefly examined. The ecological data and the information in the literature suggests that Navicula confervacea is a saprophytic species which is resistant to pollution and a stenotherm in hot water $\left(25-31^{\circ} \mathrm{C}\right)$.

\section{I. - SITUATION DE LA STATION}

Située à $100 \mathrm{~km}$ environ à l'aval de Paris (pK 105 des Services de la Navigation) et $30 \mathrm{~km}$ à l'aval de la confluence avec l'Oise, la centrale thermique E.D.F. de Porcheville comporte deux unités de quatre groupes qui totaliseront $2900 \mathrm{MW}$ en 1975 pour une consommation maximale de $110 \mathrm{~m}^{3} / \mathrm{s}$ d'eau de réfrigération puisée directement en Seine (le débit d'étiage du fleuve à Porcheville se situe autour de $\left.80 \mathrm{~m}_{\mathrm{l}}^{3} / \mathrm{s}\right)$; cette eau est déversée dans le bras de Limay, mis en communication avec la Seine pour accélérer le

1. D'après une thèse de $3^{e}$ cycle préparée au Laboratoire de Zoologie, Centre d'Hydrobiologie de l'Université de Besançon.

2. Laboratoire d'Hydroécologie, Div. Qual. Eaux, P. Pisc. C.T.G.R.E.F., Paris, Besançon. 


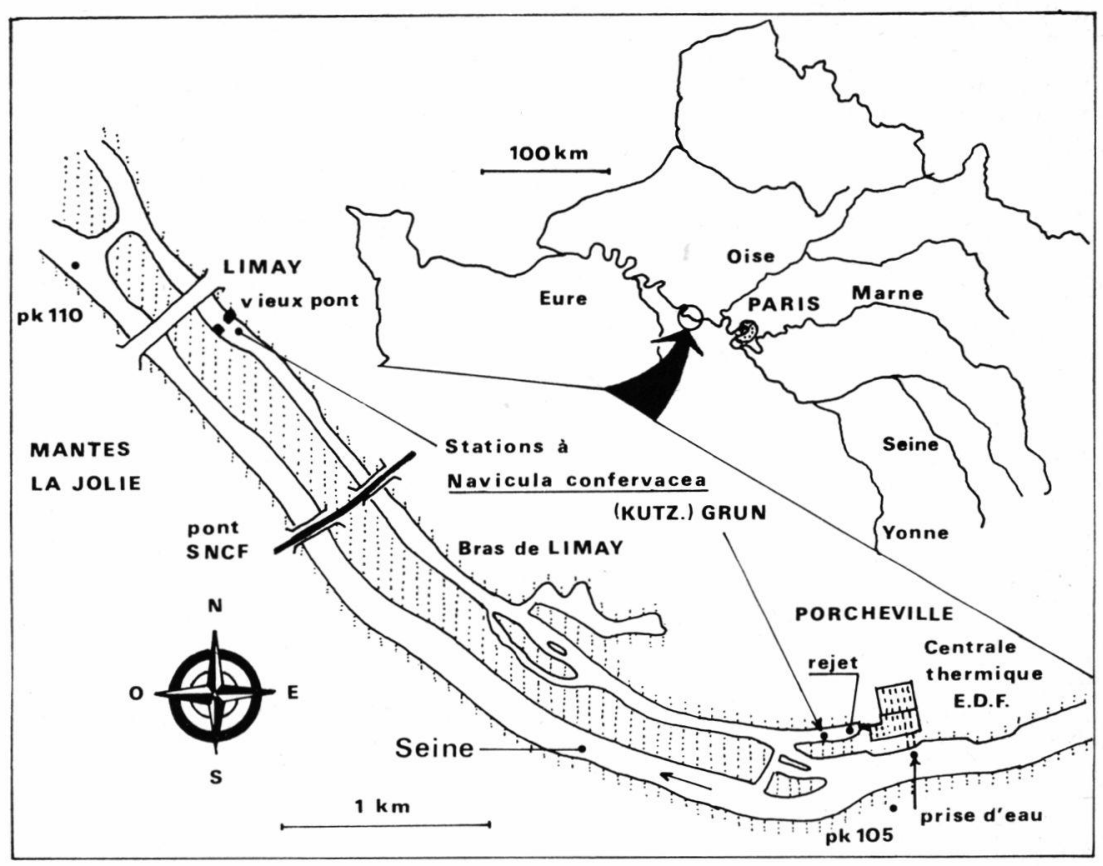

Fig. 1. - Localisation du site de Porcheville.

refroidissement. Les variations de température entre l'eau de Seine et celle du rejet de la centrale oscillent entre 5 et $7{ }^{\circ} \mathrm{C}$; par suite du fonctionnement irrégulier de certaines tranches, les variations d'amplitudes mensuelles peuvent être beaucoup plus importantes (fig. 2).

D'après Sonrel (1974), l'échauffement résiduel au niveau du pont de Limay est de 2 à $5{ }^{\circ} \mathrm{C}$.

Navicula confervacea a été trouvée en abondance au niveau du vieux pont de Limay et dans le canal de rejet de la centrale (fig. 1) durant les périodes estivales de 1972 à 1974. Elle n'a jamais été rencontrée à l'aval de la centrale thermique de Montereau où la température des eaux du rejet est souvent supérieure à $30^{\circ} \mathrm{C}$ en été mais où les eaux sont beaucoup moins polluées (Coste 1974).

\section{II. - DESCRIPTION SOMMAIRE DE L'ESPECE ET SYSTEMATIQUE}

Navicula confervacea (Kütz.) Grunow (in Van Heurck, 18801885 ) a été également décrite par Clève (1894), Hustedt (1913 in Schmidt 1930-1966). 


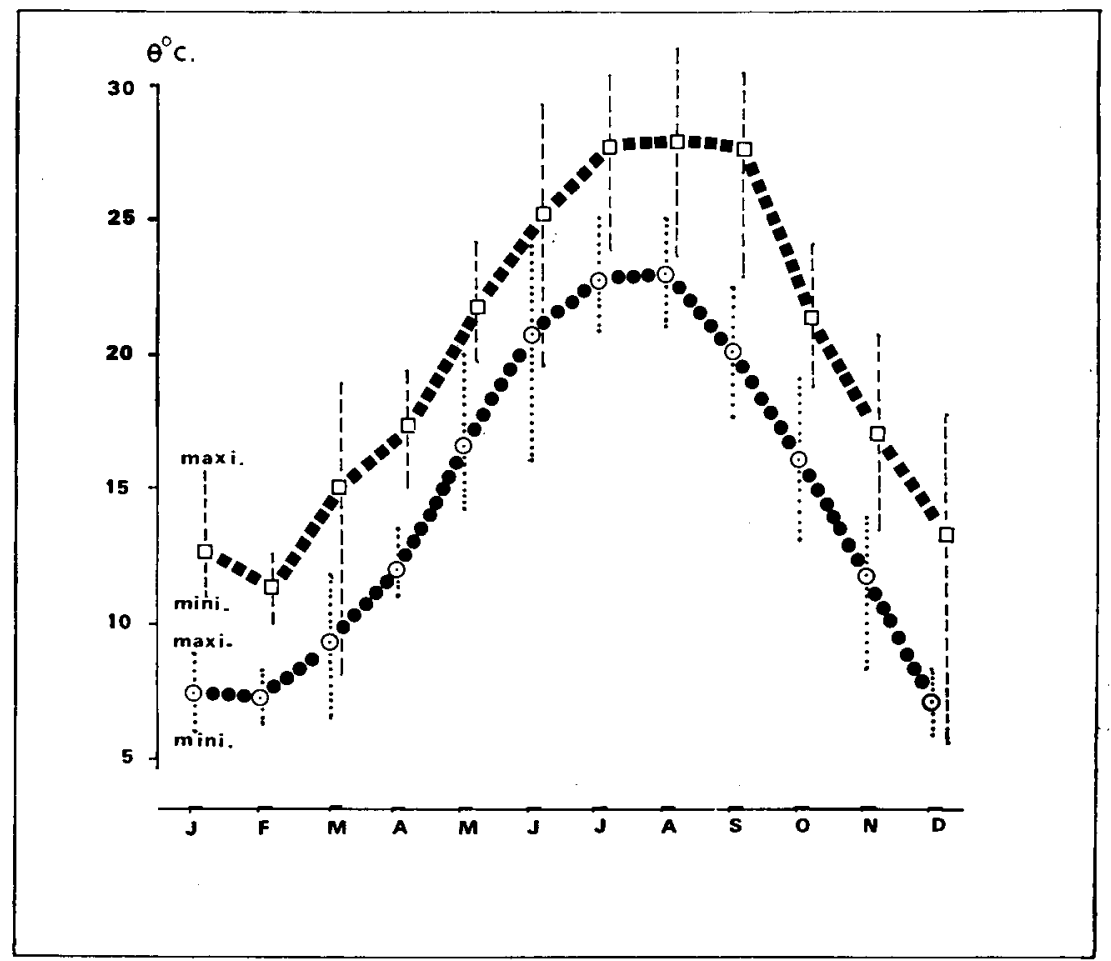

Fig. 2. - Régime thermique de la Seine à Porcheville, en 1973, au niveau de la centrale thermique E.D.F.

$\Theta^{\circ}$ moyennes mensuelles de l'eau :

O00 à l'amont de la centrale

\section{Préparations de référence}

Tempère et Peragallo, coll. diat., $2^{\circ}$ édit. (1907), $\mathrm{n}^{\circ} 289$. Collection de Clève et Möller (1877-1879), préparations $n^{\circ s} 138$ et 188.

Classée successivement dans les Entoleiae (Hustedt 1930) puis dans les Minusculae (Hustedt 1966), l'espèce a pour principaux synonymes : Diadesmis confervacea, Kützing, Diadesmis peregrina, Smith, Navicula confervacea var. peregrina, Grünow, Navicula confervacea var. hungarica, Grünow, Navicula semivirgata, Krasske, $N$. confervacea $f$. nipponica, Skvortzow, $N$. confervacea var. baikalensis, Skvortzow.

Les individus vivants observés à Porcheville et à Limay forment de longues chaînes rappelant celles des Fragilaria.

De forme elliptique lancéolée, parfois légèrement rostrée, la valve ne dépasse pas $16 \mu \mathrm{m}$ de long sur 6 à 7 de large. Le raphé, droit, court et filiforme, se termine très souvent par une boucle en forme de violon (fig. 4: photo 5) bien visible en microscopie électronique. Le nodule central est arrondi et réfringent, l'aire 
axiale lancéolée ; les stries transapicales (18 à 20 en $10 \mu \mathrm{m}$ ) légèrement radiales, apparaissent nettement ponctuées.

En vue connective (fig. 4 : photo 4 ), les cellules présentent entre elles, dans la partie médiane, un espace réfringent de forme ovoïde aplatie qui correspond à l'accolement des nodules centraux.

A

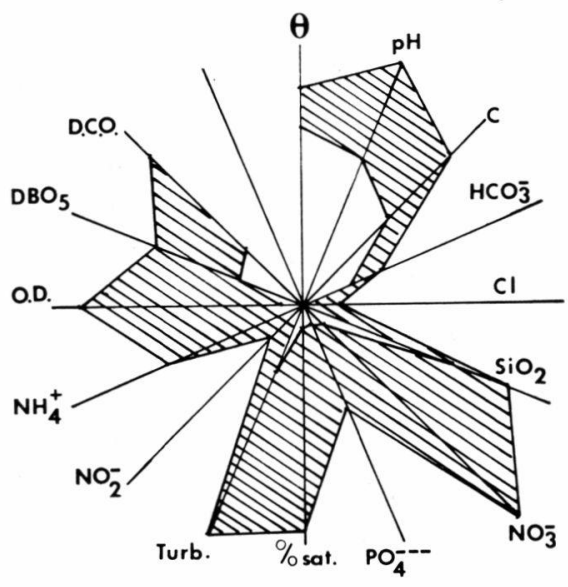

B

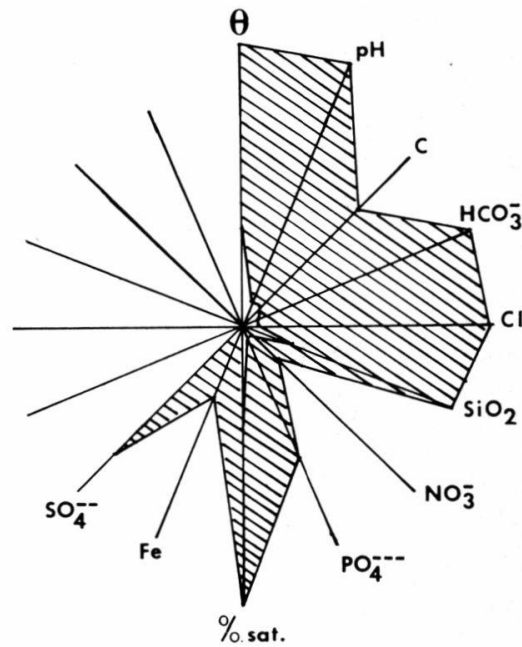

Fig. 3. - Spectres écologiques de Navicula confervacea. A : d'après les données recueillies sur la Seine; B : d'après les données de la littérature.

L'observation de l'ultrastructure au microscope électronique à balayage $^{1}$ révèle la présence de dents marginales sur le pourtour valvaire, dents du même type que celles observées chez les Fragilaria et chez la plupart des diatomées pennées formant des colonies tabulaires (Gasse 1971). Ces dents, en s'imbricant les unes dans les autres, permettent l'emboîtement des valves et la constitution des chaînes.

1. Les observations ont été réalisées sur le microscope électronique à balayage de la Faculté des Sciences d'Orsay en collaboration avec O. Roche et $\mathrm{S}$. Blaise avec l'agrément de $\mathrm{M}$. le Professeur Guinochet auxquels nous adressons nos plus vifs remerciements. 


\section{III. - REPARTITION GEOGRAPHIQUE ${ }^{2}$}

Considérée à l'origine comme endémique des régions tropicales (Clève 1894, Hustedt 1930, Krasske 1948, Bourrelly et Manguin 1952), l'espèce est également représentée dans les régions tempérées. Le tableau ci-après résume les principales stations connues à ce jour.

\section{IISTE DES STATIONS}

\section{RÉfÉRENCES}

\section{Europe}

- Allemagne :

décrite sous le nom de Navicula semivirgata en Saxe, elle a été trouvée dans les jardins botaniques de Dresde, Francfort, Gottingen et Karlsruhe dans les bassins à Victoria regia (serres chaudes).

Présente dans le delta de la Volga.

- Angleterre :

dans les bassins (Kew Gardens).

Krasske 1929-1950

Hustedt 1966.

Patrick et Reimer 1966

- France :

en Bretagne (étang du Pas du Houx); l'espèce n'a pas été trouvée vivante.

- Hongrie : Tapolcza

dans des eaux thermales $\left(t^{\circ}=30^{\circ} \mathrm{C}\right)$. Amérique

- Andes du Pérou.

-... Brésil :

sur Thucidium,

également à Rio de Janeiro.

- Equateur.

- Etats-Unis :

la plupart des Etats du Sud : Floride, Pensylvanie, Arizona, Californie, Virginie, Indiana, Kansas, etc.

Dans un affluent de la Savannah River (Lower Three runs Creek) avec une fréquence relative élevée : $38 \%$ (Caroline du Su(d).

- Guadeloupe.

- Jamaïque.

— Ile de la Trinité.

Le Cohu 1974

Grunow in Clève 1894

Manguin 1964

Krasske $1939 ; 1948 ; 1951$

Clève 1894

Clève 1894

Patrick et Reimer 1966

Reimer 1962

Bourrelly et Manguin 1952

Clève 1894

Clève, Patrick et Reimer 1966

2. Nous remercions M: le Professeur P. Bourrelly qui a bien voulu guider nos recherches et nous donner accès aux collections et à l'Herbier du Muséum National d'Histoire Naturelle. 
Asie

- Bengale : Mui How Village

dans des eaux chargées en matières organiques (prép. $\mathrm{n}^{\circ}$ 188).

- Birmanie : Rangoon.

- Formose

sur Azolla imbricata.

- Inde : Etat de Mysore (Jog Falls).

- Insulinde : Java, Bali, Sumatra.

- Japon et lac Baïkal.

- Australie : Alpes australiennes.

- Nouvelle-Calédonie :

sur racines d'Eichornia.

Clève et Müller 1877-1879

Clève 1894

Krasske 1950

Gandhi 1970

Hustedt 1937-1939

Skvortzow $1936 ; 1937$

Clève 1894

Manguin 1962

Maillard (à paraître)

- Iles Hawaii (Sandwich) : île Oahu.

Clève et Müller 1877-1879

- Iles Marquises (préparation $n^{\circ} 138$ ).

Clève et Müller 1877-1879

LISTE DES STATIONS

- Tahiti : lagon de Riewa

dans les eaux douces, saumâtres et marines.

- Nouvelle-Guinée Hollandaise. Afrique

- Afrique du Sud : provinces du Cap, du Transvaal.

- Angola.

- Congo Belge : Zaïre.

- Dahomey.

- Ethiopie : bassin inférieur de l'Aouache,

parmi les Diatomées fossiles holocènes (argiles granuleuses à Melania).

- Ghana.

- Kanem (Tchad).

- Niger.

- Sahara.

- Togo.

- Sénégal, Soudan et Mauritanie.

- Sénégal et Côte d'Ivoire.

- Tibesti et Djourab.

\section{RÉFÉRENCES}

Grunow in Clève 1894

Ricard (à paraître)

Cholnoky 1963

Cholnoky 1958; 1962; 1968

Archibald 1971

Schoeman 1973

W. et G. S. West 1897

Hustedt 1949

Zanon 1938

Hustedt 1920

Gasse 1974

Foged 1966 ; Woodhead et Tweed $1958 ; 1960$

Round 1961 ; Iltis 1972

Maillard (à paraître)

Rognon 1967

Zanon 1941

Guermeur 1954

Amosse 1970

Amosse 1934

\section{IV. - DONNEES ECOLOGIQUES}

Malgré le nombre important de stations régulièrement prospectées, l'espèce fut recensée uniquement dans le canal du rejet de la centrale thermique de Porcheville et dans son prolongement immédial (bras de Limay). 


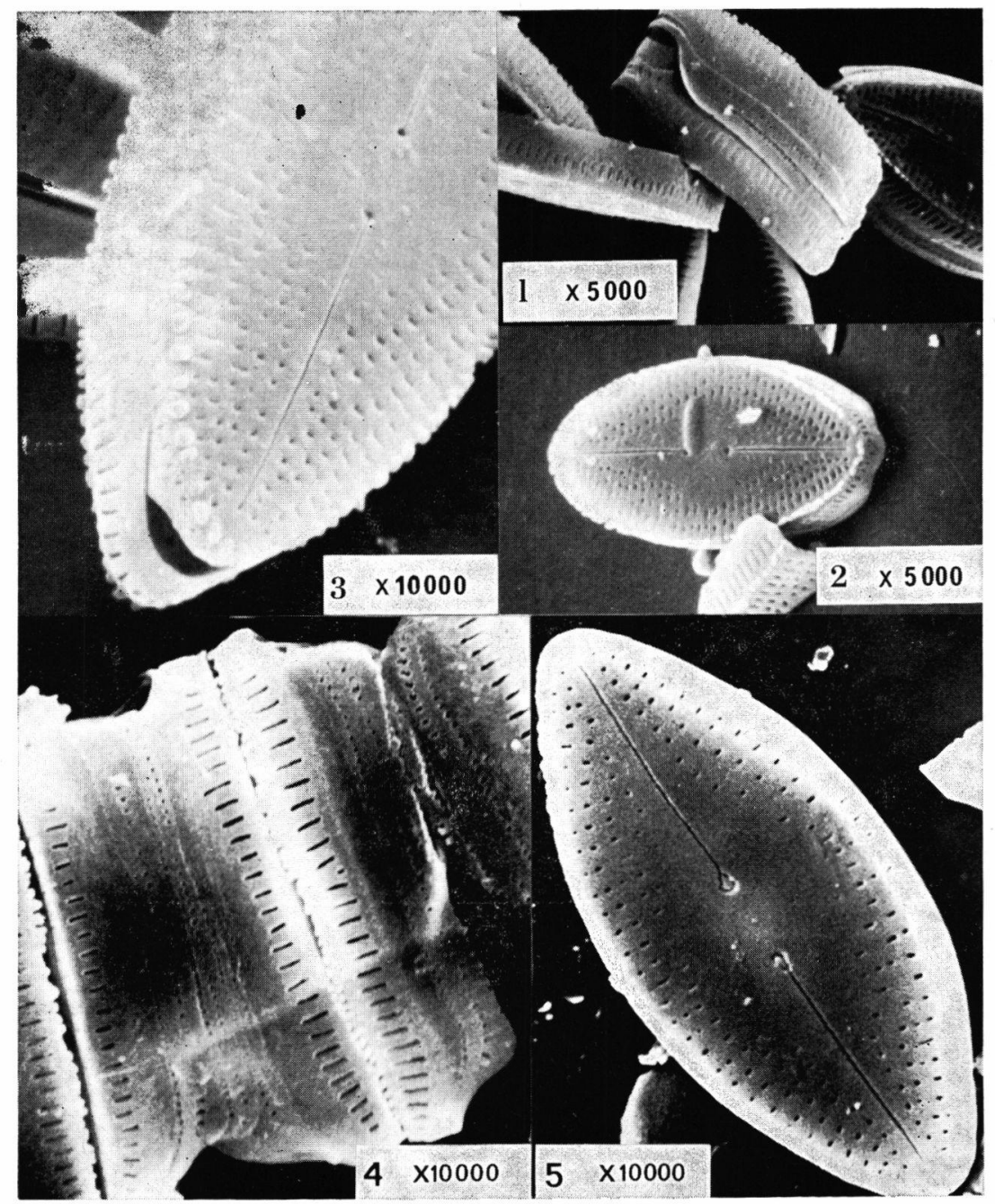

Fig. 4. - Navicula confervacea (Kütz.) Grün. Ultrastructure révélée par le microscope électronique à balayage.

Photos 1, 2, 3 : matériel provenant de Nouvelle-Calédonie, d’après F. Gasse, C.N.R.S. Bellevue.

Photos 4, 5: matériel récolté au niveau du rejet de la cenirala E.D.F. de Porcheville. Photos d'après O. Roche, C.N.R.S. Orsay. 
Les conditions très particulières régnant dans ce chenal jusqu'à Limay expliquent sans doute ce développement intense très localisé. L'espèce est présente de juillet à septembre, son abondance paraissant augmenter d'une année à l'autre. En juillet 1972, quelques rares individus font leur apparition; en 1973, l'espèce constilue $12 \%$ de la population diatomique pour atteindre $38 \%$ en juillet 1974 dans le canal de rejet et $65 \%$ dans le bras de Limay ( $3 \mathrm{~km}$ à l'aval de la centrale).

Navicula confervacea se développe indifféremment sur les galets ou sur les algues filamenteuses : Schizomeris leibleinii Kütz. (Ulvale) et Hydrodyctyon reticulatum (L.) Lagerheim (Chlorococcale), avec comme diatomées compagnes, des Centriques (Melosira ambigua, M. granulata et var., Stephanodiscus hantzschii, Cyclotella kutzingiana, C. meneghiniana) et quelques Pennées (Nitzschia palea, Navicula mutica, N. seminulum, Gomphonema parvulum...).

Remarque. - L'espèce a également été récoltée sur supports artificiels (lames de verre immergées) placés dans le rejet de la centrale.

En juillet 1974, période durant laquelle le développement optimum a été observé, la composition microfloristique est la suivante (en $\%$ de fréquence relative) :

Rejet de la centrale Bras de Limay sur galets et Schizomeris sur Hydrodyctyon

Gomphonema parvulum

2,3

1,4

Navicula confervacea

37,7

65,0

Navicula mutica

1,3

Nitzschia filiformis

1,0

Nitzschia palea

4,3

7,1

Synedra ulna

5,1

4,0

Diatomées centriques

38,1

17,2

Autres espèces

9,4

3,5

La plupart des espèces citées précédemment affectionnent les milieux fortement pollués. Les rejets de l'agglomération parisienne, ceux de la station d'épuration d'Achères, la présence d'industries chimiques sur l'Oise et à l'aval de Paris, contribuent à la détérioration de la qualité de l'eau au niveau de Porcheville. Les variations des principaux paramètres physico-chimiques observées durant une année sont les suivantes : 
Tabieau II1. - Composition physico-chimique de l'eau (valeurs extrêmes) au niveau de Porcheville et données bibliographiques.

\begin{tabular}{|c|c|c|c|c|c|c|}
\hline & \multirow{2}{*}{\multicolumn{2}{|c|}{$\begin{array}{l}\text { Amplitude } \\
\text { des } \\
\text { variations } \\
\text { durant } \\
\text { l'année }\end{array}$}} & \multicolumn{2}{|c|}{$\begin{array}{l}\text { Valeurs } \\
\text { relevées } \\
\text { lors du déve- } \\
\text { loppement } \\
\text { optimum } \\
\text { (fin juillet } \\
1974 \text { ) }\end{array}$} & \multirow{2}{*}{\multicolumn{2}{|c|}{$\begin{array}{c}\text { Données } \\
\text { de la } \\
\text { bibliographie } \\
\text { in Reimer } \\
\text { (1962) }\end{array}$}} \\
\hline & & & $\begin{array}{l}\text { rejet } \\
\text { E.D.F. }\end{array}$ & $\begin{array}{l}\text { bras cle } \\
\text { Limay }\end{array}$ & & \\
\hline Température $\left({ }^{\circ} \mathrm{C}\right)$ & $8-$ & -31 & 28 & 25 & 15 & -40 \\
\hline $\mathrm{pH}$ & 6,2 & $-\quad 7,7$ & 7,7 & 7,6 & 4,0 & $-\quad 8,2$ \\
\hline \multicolumn{7}{|l|}{ Conductivité } \\
\hline $\begin{array}{l}\text { en } \mu \mathrm{mhos} / \mathrm{cm} / \mathrm{cm}^{2} \\
\text { Turbidité }\end{array}$ & $\begin{array}{r}420 \\
10\end{array}$ & $\begin{array}{l}-710 \\
-35\end{array}$ & $\begin{array}{r}614 \\
35\end{array}$ & $\begin{array}{r}612 \\
20\end{array}$ & 87 & $\begin{array}{l}-580 \\
--\end{array}$ \\
\hline Oxygène dissous en $\mathrm{mg} / \mathrm{l}$ & 0,4 & $-\quad 9,4$ & 0,8 & 3,1 & - & $-\cdots$ \\
\hline$\%$ de saturation & 10 & -80 & 19 & 38 & 40 & -100 \\
\hline Alcalinité totale en $\mathrm{HCO}_{3}^{-}$ & 225 & -275 & - & - & 12 & -915 \\
\hline Phosphates $\left(\mathrm{PO}_{4}^{---}\right)$ & 0,49 & $9-\quad 1,7$ & - & - & 0,002 & $2, \quad 2,06$ \\
\hline Sels ammoniacaux $\left(\mathrm{NH}_{4}^{+}\right)$ & 1,5 & $-8,5$ & - & - & $\ldots$ & -- \\
\hline Nitrites $\left(\mathrm{NO}_{2}^{-}\right)$ & 0,1 & $-\quad 1,3$ & - & - & - & -- \\
\hline Nitrates $\left(\mathrm{NO}_{3}^{-{ }^{-}}\right)$ & 1,0 & $-13,5$ & - & - & 0,02 & $-\quad 2,81$ \\
\hline Chlorures (Cl) & 18 & -- & - & - & 5 & -180 \\
\hline $\mathrm{SiO}_{2} \mathrm{ppm}$ & - & -- & 12,2 & 10,6 & 3,0 & $-12,7$ \\
\hline Fe ppm & - & -- & - & 一 & 0,001 & 1. 0,116 \\
\hline $\mathrm{SO}_{4} \mathrm{ppm}$ & - & -- & - & -- & 1 & -260 \\
\hline $\mathrm{DBO}_{6}$ & 10 & -22 & - & - & - & -- \\
\hline DCO & 28 & -75 & - & - & - & -- \\
\hline $\begin{array}{l}\text { Détergents anioniques } \\
\text { en TBS }\end{array}$ & 0,1 & $-\quad 0,7$ & - & - & - & -- \\
\hline
\end{tabular}

Remarque. - Le dosage des métaux lourds effectué sur les matières en suspension et le sédiment entre mai 1972 et juillet 1974, fait apparaître des teneurs légèrement inférieures à celles observées dans la Seine au niveau de l'agglomération parisienne, comme l'indiquent les résultats suivants exprimés en ppm de matière sèche.

1. Les analyses physico-chimiques classiques et les dosages de métaux nous ont été communiqués par $M$. Nisbet et $D$. Lintignat, laboratoire de Chimie des Eaux et des Micropolluants, Div. Qual. Eaux, C.T.G.R.E.F., Paris. 
Tableau III1. - Valeurs comparées des teneurs en métaux lourds relevées dans la Seine (sur les matières en suspension et le sédiment) au niveau de Paris et de Porcheville. Résultats exprimés en ppm de matière sèche.

\begin{tabular}{lcc}
\hline & au niveau & au niveau \\
de Paris & de Porcheville \\
Plomb & $130-2450$ & $265-500$ \\
Cuivre & $190-1790$ & $135-905$ \\
Chrome & $110-1330$ & $330-640$ \\
Cadmium & $14,1-106$ & $30,5-60,5$ \\
Nickel & $44-258$ & $112-208$ \\
Zinc & $115-5130$ & $100-2600$ \\
\hline
\end{tabular}

Navicula confervacea atteint son oplimum de développement en juillet et en août, durant la période qui correspond à l'étiage ; elle supporte donc les conditions les plus sévères : température élevées $\left(25\right.$ à $30^{\circ} \mathrm{C}$ ), déficit en oxygène dissous important (le pourcentage de saturation est parfois inférieur à $10 \%$ ), teneurs en sels dissous élevées. L'espèce n'a jamais été trouvée en période hivernale.

La prolifération est de moindre importance dans le rejet immédiat que dans le bras de Limay. Les fortes turbidités pouvant constituer un facteur limitant.

Les valeurs obtenues à Porcheville, comparées à celles recueillies par Reimer (1962), Bourrelly et Manguin (1952), Hustedt (19371939), Cholnoky (1958), permettent d'ébaucher la définition du spectre écologique de l'espèce (fig. 3), selon le procédé utilisé par Verneaux (1973). On constate que les pourcentages de fréquence relative relevés à Porcheville, comme les valeurs de la conductivité, du déficit en oxygène et les teneurs en nitrates sont beaucoup plus élevés que ceux observés par les auteurs précédents.

Des mesures effectuées par E.D.F. ont montré que l'oxygène dissous provient essentiellement de l'activité photosynthétique du phytoplancton, les teneurs devenant pratiquement nulles la nuit; Navicula confervacea se comporte donc, dans ces milieux, en saprophyte.

Les données autoécologiques paraissent, comme c'est souvent le cas, relativement contradictoires. Selon Patrick (1966), il s'agit d'une espèce aérophile préférant les eaux peu profondes susceptibles de s'échauffer ; pour Reimer (1962), elle affectionne les eaux calmes, bien oxygénées, pauvres en matières organiques, parfois dystrophes, le $\mathrm{pH}$ optimum se situant près de la neutralité. Reimer considère l'espèce comme eurytherme (15 à $\left.40^{\circ} \mathrm{C}\right)$.

1. Les analyses physico-chimiques classiques et les dosages de métaux nous ont été communiqués par M. Nisbet et D. Lintignat, laboratoire de Chimie des Faux et des Micropolluants, Div. Qual, Eaux, C.T.G.R.E.F., Paris. 
Selon Hustedt (1937), l'espèce, trouvée dominante en Insulinde dans $20 \%$ des récoltes, serait oligohalobe (voire indifférente), euryionique (pH 5 à 8,2), avec un $\mathrm{pH}$ optimum compris entre 7 et 7,5 , euryoxybionte, saprophyte lorsqu'elle est dominante et devrait être classée comme $\beta$ mésosaprobe.

Pour Manguin (1952, 1962, 1964), il s'agit d'une espèce « endémique des régions tropicales » largement répandue en Guadeloupe où elle recherche plus particulièrement les biotopes aérés ( $\mathrm{pH} 6,0$ à 7,5$)$. En Nouvelle-Calédonie, elle affectionne les petites collections d'caux riches en matière organique végétale el animale. Cholnoky (1958, 1968) considère celte espèce comme « indicatrice» d'eau polluée par des matières organiques azotées, capable de supporter des déficits en $\mathrm{G}_{2}$ dissous important. Son abondance dans la rivière Jukskei ainsi qu'à l'aval de la station d'épuration de Pretoria où elle est associée à Gomphonema parvulum et Navicula seminulum, est liée à l'instabilité du milieu et à l'enrichissement en azote dissous. En Afrique du Sud, le pH optimum se situerait selon l'auteur autour de 8,4 ; l'abondance de Navicula confervacea en Europe, parmi les plantes exotiques, s'expliquerait par l'adjonction d'engrais azotés ou de purin dans les collections d'eau. D'après Cholnoky, l'espèce serait $\mathrm{N}$-hétérotrophe facultative comme Navicula seminulum qui lui est fréquemment associée (à Porcheville notamment) ; elle aurait en outre la propriété de désaminer rapidement le glycocolle et l'alanine.

Schoeman (1973) considère, à juste titre semble-t-il, cette espèce comme un excellent «indicateur » des pollutions organiques azotées intermittentes.

\section{IV. - CONCLUSION}

La prolifération de Navicula confervacea à Porcheville, dans un milieu fortement pollué, où les températures moyennes mensuelles ne descendent pas en dessous de $11^{\circ} \mathrm{C}$ mais restent supéricures à $25^{\circ} \mathrm{C}$ pendant 4 mois de l'année avec des maxima supérieurs à $30^{\circ} \mathrm{C}$, semble relativement en accord avec les données recueillies par Cholnoky. En conséquence, il paraît raisonnable d'attribuer une telle prolifération à la juxtaposition d'un effet thermique marqué et d'une pollution organique importante, la température élevée et les fortes teneurs en sels d'azote dissous contituant sans doute les paramètres déterminants. 


\section{TRAVAUX GITÉS}

Amosse (A.). 1934. - Diatomées du Tibesti et du Djourab. Mém. Acad. Sci. Inst. Fr., 61 (2 sér.) : 143-153.

Amosse (A.). 1970. - Diatomées marines et saumâtres du Sénégal et de la Côte d'Ivoire. Bull. Inst. Fr. Afr. noire, 32 (2-A) : 289-311.

Archibald (R. E. M.). 1971. - Diatoms from the Vall Dam Catchment Area Transvaal, South Africa. Botanica mar., $14: 17-70$.

Bourrelay (P.) et Manguin (E.). 1952. - Algues d'eau douce de la Guadeloupe et dépendances recueillies par la mission ALLORGE 1936. Sedes, Paris : 281 p.

Cholnoky (B. J.). 1954. - Hydrobiologische Untersuchungen in Transvaal II. Selbstreinigung im Jukskei-Crocodile Flussystem. Hydrobiologia, 11 : 215-266.

Cholnoky (B. J.). 1962. - Beiträge zur Kenntnis der ökologie der Diatomeen in Ost-Transvaal. Hydrobiologia, 19 : 57-119.

Cholnoky (B. J.). 1963. - Ein Beitrag zur Kenntnis der Diatomeenflora von Holländisch Neu-Guinea. Nova Hedwigia, 5 : 157-198.

Cholvoky (B. J.). 1968. - Die Ökologie der Dialomeen in Binnengewässer. Cramer ed. : $699 \mathrm{p}$.

CuÈve (P. T.). 1894. - Synopsis of the naviculoid diatoms. K. svenska Vetensk-Akad. Handl., 26 (1) : 1-194.

Coste (M.). 1974. - Etudes sur la mise au point d'une méthode biologique de détermination de la qualité des eaux en milieu fluvial. Trav. Div, Qual. Eaux, P. Pisc. C.T.G.R.E.F., ronéo : 79 p.

Foged (N.). 1966. - Freshwater diatoms from Ghana. Biol. Skr. Dan. Vid. Selsk., 15. (1) : $169 \mathrm{p}$.

GaNdHI (H. P.). 1970. - A further contribution to the diatomflora of the Jog-Falls; Mysore State, India. In Diatomaceae II. Beih. $z$. Nova Hedwigia, 31 : 757-813.

Gasse (F.). 1971. - Organisation structurale des colonies de quelques diatomées pennées araphidées révélée par le microscope électronique à balayage. C. R. Acad. Sc. Paris, sér. D, 272 : 3169-3172.

Gasse (F.). 1974. - Les diatomées holocènes du bassin inférieur de l'Aouache (dépression des Danakil, Ethiopie). Leur signification paléo-écologique. Int. Revue Ges. Hydrobiol., 59 (1) : 123-146.

Guermeur (P.). 1954. - Diatomées de l'A.O.F. (première liste - Sénégal). Cat. Inst. fr. Afr. noire, $12: 1-137$.

Hustedt (F.). 1910. - Beitrag zur Algenflora von Afrika. Bacillariales aus Dahomé. Arch. Hydrobiol., 6 : 307-346.

Hustedt (F.). 1930. - Bacillariophyta. Pascher's Susswasserflora Mitteleuropas, $10: 1-468$.

Husted (F.). 1937-1939. — Systematische und ökologische Untersuchungen über die Diatomeen-Flora von Java, Bali und Sumatra nach dem material der Deutschen Limnologischen Sunda-Expedition (1), Systematischer Teil. Arch. Hydrobiol., suppl., 15 : 131506.

HustedT (F.). 1949. - Süsswasser-diatomeen aus dem Albert-National Park in Belgisch-Kongo. Expl. der Park Nat. Albert, Miss. H. Damas (1935-1936), $8: 1-199$.

Hustedt (F.). 1961-1966. - Die Kieselalgen. Rabenhorst Kryplogamenflora, 7 (3) : $1-816$.

Iltis (A.). 1972. - Algues des eaux natronées du Kanem (Tchad), $1^{\text {re }}$ partie. Cah. O.R.S.T.O.M., sér. Hydrobiol., 6 (3-4) : 173-246.

Krasske (G.). 1929. - Beiträge zur Kenntnis der Diatomeenflora Sachsens. Botan. Arch., $27: 348-380$. 
Krasske (G.). 1939. - Zur Kieselalgenflora Brasiliens I. Arch. Hybrobiol., 35 : $552-562$.

Krasske (G.). 1948. - Diatomeentropischer Moosrasen. Svensk. Bot. Tidskr., $42: 404-443$.

Krasske (G.). 1950. - Diatomeas del Archipiélago de Formosa (Taiwan). Bol. Soc. Biol. Concepcion, 25 : 75-107.

Krasske (G.). 1951. - Die Diatomeenflora des Açudas Nordostbrasiliens. Arch. Hydrobiol., 44 : 639-653.

LE CoHu (R.). 1974. - Recherches expérimentales sur l'écologie des algues d'eau douce. Utilisation des enceintes en milieu naturel. Thèse Doctorat Sci. Rennes : 271 p.

Manguin (E.). 1962, - Contribution à la connaissance de la flore diatomique de la Nouvelle-Calédonie. Mém. Mus, natn. Hist. nat. Paris, sér. B, Bot., 12 (1) : 1-40.

Manguin (E.). 1964. - Contribution à la connaissance de la flore diatomique des Andes du Pérou. Mém. Mus. natn. Hist. nat. Paris, sér. B, Bot., 12 (2) : 41-98.

Patrick (R.) et Reimer (C.). 1966. - The Diatoms of the United States. Mon. Acad. Nat. Sci. Philad., 13:688 p.

Reimer (C. W.). 1962. - Diatoms and their physico-chemical environment. Environmental Health series. Water Supply and Pollution Control. 3th Seminar: 19-28.

Rognon (P.). 1967. - Le massif de l'Atakor et ses bordures (Sahara central). Etude géomorphologique. Cent. Nat. Rech. Zones arides. C.N.R.S., Paris : 560 p.

Round (F. E.). 1961. - The composition of some diatomites from the Southern Sahara. Jl. R. microsc. Soc., $80: 59-69$.

Sснміdт (A.). 1874-1944. - Atlas der Diatomaceen-Kunde. Fortges. v. M. Schmidt, F. Fricke, O. Müller, H. Heiden, F. Hustedt. Reprint 1972, O. Koeltz : 1-480.

Schoeman (F. R.). 1973. - A systematical and ecological study of the diatom flora of Lesotho with special reference to the water quality. Pretoria : 355 p.

Skvortzov (B. W.). 1936. Diatoms from Kizaki lake, Honshu Island. Nippon. Phil. Journ. Sci., 61 (1) : 9-73.

Skvortzov (B. W.). 1937, - Bottom diatoms from Olhon Gate of Baïkal lake, Siberia. Nippon. Phil. Journ. Sci., 62 (3) : 293-377.

Sonrel (J.). 1974. - Influence de la centrale de Porcheville sur la qualité de l'eau de Seine. T.S.M. l'Eau, 8-9: 459-465.

Van Heurck (H.). 1880-1885. - Synopsis des dialomées de Belgique. Anvers : $235 \mathrm{p}$.

Verneaux (J.). 1973. - Recherches écologiques sur le réseau hydrographique $d u$ Doubs. Essai de biotypologie. Thèse Doctorat d'État, Sci. Nat., Univ. Besançon, 84, C.N.R.S., A.O. 8823 : 260 p.

West (W.) et West (G. S.). 1897. - Welwitsch's African Freshwater Algae. J. Bot., $35: 1$ et suiv.

Woodhead (N.) et Tweed (R. D.). 1958. - A check list of tropical West African algae (fresh and brackish water). Hydrobiologia, 11 : 299-395.

Woodhead (N.) et TweEd (R. D.). 1960. - A second check list of tropical West African algae. Hydrobiologia, 15 (3) : 225-286.

Zanon (D. V.). 1938. - Diatomee della Regione del Kivu (Congo Belga). Commentat. pontif. Acad. Scient., 2 : 535-668.

Zanon (D. V.). 1941. - Diatomee dell'Africa occidentale francese. Commentat. pontif. Acad. Scient., 5 : 1-60. 